\title{
Atopic Dermatitis: Evolution and Revolution in Therapy
}

\author{
Margaret M. Appiah, BS; Michael A. Haft, BS; Lawrence F. Eichenfield, MD
}

$\Lambda$ topic dermatitis (AD) is an incredibly common chronic skin disease, affecting up to $25 \%$ of children and $7 \%$ of adults in the United States. ${ }^{1,2}$ Despite the prevalence of this disease and its impact on patient quality of life, research and scholarly work in $\mathrm{AD}$ has been limited until recent years. A PubMed search of articles indexed for MEDLINE using the term atopic dermatitis showed that there were fewer than 500 articles published in 2000 and 965 in 2010; with our more recent acceleration in research, there were 2168 articles published in 2020 and more than 1300 published in just the first half of 2021 (through June). This new research includes insights into the pathogenesis of $\mathrm{AD}$ and study of the disease impact and comorbidities as well as an extensive amount of drug development and clinical trial work for new topical and systemic therapies.

\section{New Agents to Treat AD}

The 2016 approval of crisaborole, ${ }^{3}$ a phosphodiesterase 4 inhibitor, followed by the approval of dupilumab, an IL-4 and IL-13 pathway inhibitor and the first biologic agent approved for $\mathrm{AD}_{,}{ }^{4}$ ushered in a new age of therapy. We currently are awaiting the incorporation of a new set of topical nonsteroidal agents, oral Janus kinase (JAK) inhibitors, and new biologic agents for $\mathrm{AD}$, several of which have completed phase 3 trials and extended safety evaluations. How these new drugs will impact our standard treatment across the spectrum of care for $\mathrm{AD}$ is not yet known.

The emergence of new systemic therapies is timely, as the most used systemic medications previously were oral corticosteroids, despite their use being advised against in standard practice guidelines. Other agents such as methotrexate, cyclosporine, azathioprine, and mycophenolate are discussed in the literature and $\mathrm{AD}$ treatment guidelines as being potentially useful, though absence of US Food and Drug Administration (FDA) approval and the need for frequent laboratory monitoring, as well as drug-specific side effects and an increased risk of infection, limit their use in the United States, especially in pediatric and adolescent populations. ${ }^{5}$

The approval of dupilumab as a systemic therapyinitially for adults and subsequently for teenagers (12-17 years of age) and then children (6-11 years of age) - has markedly influenced the standard of care for moderate to severe AD. This agent has been shown to have a considerable impact on disease severity and quality of life, with a good safety profile and the added benefit of not requiring continuous (or any) laboratory monitoring. ${ }^{6-8}$ Ongoing studies of dupilumab in children (ClinicalTrials. gov identifiers NCT02612454, NCT03346434), including those younger than 1 year, ${ }^{9}$ raise the question of how

From the Departments of Dermatology and Pediatrics, University of California San Diego. Ms. Appiah and Dr. Eichenfield also are from Rady Children's Hospital San Diego. Mr. Haft also is from the University of Rochester School of Medicine, New York.

Ms. Appiah and Mr. Haft report no conflict of interest. Dr. Eichenfield has served as an adviser, consultant, and/or clinical study investigator for AbbVie; Almirall; Amgen; Arcutis Biotherapeutics; Arena Pharmaceuticals; Dermavant Sciences, Inc; Dermira, Inc; Eli Lilly and Company; Galderma; Glenmark Pharmaceuticals/Ichnos Sciences, Inc; Incyte Corporation; Laboratoires Forté Pharma; LEO Pharma; Novartis; Ortho Dermatologics; Pfizer; Regeneron Pharmaceuticals; and Sanofi Genzyme.

Correspondence: Lawrence F. Eichenfield, MD, Pediatric and Adolescent Dermatology, Rady Children's Hospital-San Diego, 3020 Children's Way, Mail Code 5092, San Diego, CA 92123 (leichenfield@ucsd.edu).

doi: 10.12788 /cutis.0337 
commonly this medication might be incorporated into care across the entire age spectrum of patients with AD. What standards will there be for assessment of severity, disease impact, and persistence to warrant use in younger ages? Will early treatment with novel systemic agents change the overall course of the disease and minimize the development of comorbidities? The answers to these questions remain to be seen.

$J A K$ Inhibitors for $A D$-Additional novel therapeutics currently are undergoing studies for treatment of $\mathrm{AD}$, most notably the oral JAK inhibitors upadacitinib, ${ }^{10}$ baricitinib, ${ }^{11}$ and abrocitinib. ${ }^{12}$ Each of these agents has completed phase 3 trials for AD. Two of these agentsupadacitinib and baricitinib-have prior FDA approval for use in other disease states. Of note, baricitinib is already approved for treatment of moderate to severe $\mathrm{AD}$ in adults in more than 40 countries $^{13}$; however, the use of these agents in other diseases brings about concerns of malignancy, severe infection, and thrombosis. In the clinical trials for $\mathrm{AD}$, many of these events have not been seen, but the number of patients treated is limited, and longer-term safety assessment is important.10,11

How will the oral JAK inhibitors be incorporated into care compared to biologic agents such as dupilumab? Tolerance and more serious potential adverse events are concerns, with nausea, headaches, and acneform eruptions being associated with some of the medications, in addition to potential issues with herpes simplex and zoster infections. However, oral JAK inhibitors have the benefit of not requiring injections, something that many patients may prefer, and data show that these drugs generally are associated with a rapid reduction in pruritus and, depending on the drug, very quick and profound effects on objective signs of AD. ${ }^{10-12}$ Two head-to-head studies have been completed comparing dupilumab to oral JAK inhibitors in adults: the JADE COMPARE trial examining dupilumab vs abrocitinib ${ }^{12}$ and the Heads UP trial comparing dupilumab vs upadacitinib. ${ }^{14}$ Compared to dupilumab, higher-dose abrocitinib showed more rapid responses, superiority in itch response, and similarity or superiority in other outcomes depending on the time point of the evaluation. Adverse event profiles differed; for example, abrocitinib was associated with more nausea, acneform eruptions, and herpes zoster, while dupilumab had higher rates of conjunctivitis. ${ }^{12}$ Upadacitinib, which was only studied at higher dosing (30 mg daily), showed superiority to dupilumab in itch response and in improvement in $\mathrm{AD}$ severity in multiple outcome measures; however, there were increases in serious infections, eczema herpeticum, herpes zoster, and laboratory-related adverse events..$^{14}$ Dupilumab has the advantage of studies of extended use along with real-world experience, generally with excellent safety and tolerance other than injection-site reactions and conjunctivitis. ${ }^{8}$ Biologics targeting IL-13-tralokinumab and lebrikizumab-also are to be added to our armamentarium. ${ }^{15,16}$ The addition of these agents and JAK inhibitors as new systemic treatment options points to the quickly evolving future of $\mathrm{AD}$ treatment for patients with extensive disease.

New topical therapies in development provide even more treatment options. New nonsteroidal topicals include topical JAK inhibitors such as ruxolitinib ${ }^{17}$; tapinarof, ${ }^{18}$ an aryl hydrocarbon receptor modulator; and phosphodiesterase 4 inhibitors. These agents may be useful either as monotherapy, as studied, potentially without the regional limitations associated with stronger topical corticosteroids, but also should be useful in clinical practice as part of therapeutic regimens with other topical steroid and nonsteroidal agents.

\section{The Microbiome and AD}

In addition, research looking at topical microbes as specific interventions that may mediate the microbiome and inflammation of $\mathrm{AD}$ are intriguing. A recent phase 1 trial from the University of California San Diego ${ }^{19}$ indicated that topical bacteriotherapy directed at decreasing Staphylococcus aureus may provide an impact in AD. Observations by Kong et $\mathrm{al}^{20}$ showed that gram-negative microbiome differences are seen in $\mathrm{AD}$ patients compared to unaffected individuals, which has fueled studies showing that Roseomonas mucosa, a gram-negative skin commensal, when applied as a topical live biotherapeutic agent has improved disease severity in children and adults with AD. ${ }^{21}$ Although further studies are underway, these initial data suggest a role for microbiomemodifying therapies as $\mathrm{AD}$ treatment.

\section{Chronic Hand Eczema}

Chronic hand eczema (CHE), which has considerable overlap with $\mathrm{AD}$ in many patients, especially children and adolescents, ${ }^{22-24}$ is another area of interesting research. This high-prevalence condition is associated with allergic and irritant contact dermatitis ${ }^{24-26}$ - conditions that are both considered alternative diagnoses for and exacerbators of $\mathrm{AD}^{27}$ - and is a disease process currently being targeted for new therapies. Delgocitinib (NCT04872101, NCT04871711), the novel JAK inhibitor ARQ-252 (NCT04378569), among other topical agents, as well as systemic therapeutics such as gusacitinib (NCT03728504), are in active trials for CHE. Given CHE's impact on quality of life ${ }^{28}$ and its overlap with $\mathrm{AD}$, investigation into this disorder can help drive future AD research as well as lead to better knowledge and treatment of CHE.

\section{Final Thoughts}

Despite the promising results of these myriad new therapies in $\mathrm{AD}$, there are many factors that influence how and when we use these drugs, including their approval status, FDA labeling, and the ability of patients to access and afford treatment. Additionally, continued study is needed to evaluate the long-term safety and extended efficacy of newer drugs, such as the oral JAK inhibitors. Despite these hurdles, the current landscape of research 
and development is rapidly evolving. Compared to the many years when only one main group of therapies was a reasonable option for patients, the future of $\mathrm{AD}$ treatment looks bright.

\section{REFERENCES}

1. Eichenfield LF, Tom WL, Chamlin SL, et al. Guidelines of care for the management of atopic dermatitis: section 1. diagnosis and assessment of atopic dermatitis. J Am Acad Dermatol. 2014;70:338-351. doi:10.1016/j .jaad.2013.10.010

2. Chiesa Fuxench ZC, Block JK, Boguniewicz M, et al. Atopic dermatitis in America study: a cross-sectional study examining the prevalence and disease burden of atopic dermatitis in the US adult population. J Invest Dermatol. 2019;139:583-590. doi:10.1016/j.jid.2018.08.028

3. FDA approves Eucrisa for eczema. News release. US Food and Drug Administration; December 14, 2016. Accessed August 16, 2021. https://www.fda.gov/news-events/press-announcements/ fda-approves-eucrisa-eczema

4. Gooderham MJ, Hong HC, Eshtiaghi P, et al. Dupilumab: a review of its use in the treatment of atopic dermatitis. J Am Acad Dermatol. 2018;78 (3 suppl 1):S28-S36. doi:10.1016/j.jaad.2017.12.022

5. Sidbury R, Davis DM, Cohen DE, et al. Guidelines of care for the management of atopic dermatitis: section 3. management and treatment with phototherapy and systemic agents. J Am Acad Dermatol. 2014;71:327-349. doi:10.1016/j.jaad.2014.03.030

6. Paller AS, Siegfried EC, Thaçi D, et al. Efficacy and safety of dupilumab with concomitant topical corticosteroids in children 6 to 11 years old with severe atopic dermatitis: a randomized, double-blinded, placebocontrolled phase 3 trial. J Am Acad Dermatol. 2020;83:1282-1293. doi:10.1016/j.jaad.2020.06.054

7. Simpson EL, Paller AS, Siegfried EC, et al. Efficacy and safety of dupilumab in adolescents with uncontrolled moderate to severe atopic dermatitis: a phase 3 randomized clinical trial. JAMA Dermatol. 2020;156:44-56. doi:10.1001/jamadermatol.2019.3336

8. Deleuran M, Thaçi D, Beck LA, et al. Dupilumab shows long-term safety and efficacy in patients with moderate to severe atopic dermatitis enrolled in a phase 3 open-label extension study. J Am Acad Dermatol. 2020;82:377-388. doi:10.1016/j.jaad.2019.07.074

9. Paller AS, Siegfried EC, Simpson EL, et al. A phase 2, open-label study of single-dose dupilumab in children aged 6 months to $<6$ years with severe uncontrolled atopic dermatitis: pharmacokinetics, safety and efficacy. J Eur Acad Dermatol Venereol. 2021;35:464-475. doi: 10.1111/jdv.16928

10. Reich K, Teixeira HD, de Bruin-Weller M, et al. Safety and efficacy of upadacitinib in combination with topical corticosteroids in adolescents and adults with moderate-to-severe atopic dermatitis (AD Up): results from a randomised, double-blind, placebo-controlled, phase 3 trial. Lancet. 2021;397:2169-2181. doi:10.1016/S0140-6736(21)00589-4

11. Simpson EL, Forman S, Silverberg JI, et al. Baricitinib in patients with moderate-to-severe atopic dermatitis: results from a randomized monotherapy phase 3 trial in the United States and Canada (BREEZE-AD5). J Am Acad Dermatol. 2021;85:62-70. doi:10.1016/j.jaad.2021.02.028

12. Bieber T, Simpson EL, Silverberg JI, et al. Abrocitinib versus placebo or dupilumab for atopic dermatitis. N Engl J Med. 2021;384:1101-1112. doi:10.1056/NEJMoa2019380

13. Lilly and Incyte provide update on supplemental New Drug Application for baricitinib for the treatment of moderate to severe atopic dermatitis. News release. Eli Lilly and Company; July 16, 2021. Accessed August 16, 2021. https://investor.lilly.com/news-releases /news-release-details/lilly-and-incyte-provide-update-supplemental new-drug
14. Blauvelt A, Teixeira HD, Simpson EL, et al. Efficacy and safety of upadacitinib vs dupilumab in adults with moderate-to-severe atopic dermatitis: a randomized clinical trial [published online August 4, 2021]. JAMA Dermatol. doi:10.1001/jamadermatol.2021.3023

15. Guttman-Yassky E, Blauvelt A, Eichenfield LF, et al. Efficacy and safety of lebrikizumab, a high-affinity interleukin 13 inhibitor, in adults with moderate to severe atopic dermatitis: a phase $2 b$ randomized clinical trial. JAMA Dermatol. 2020;156:411-420. doi:10.1001 /jamadermatol.2020.0079

16. Silverberg JI, Toth D, Bieber $\mathrm{T}$, et al. Tralokinumab plus topical corticosteroids for the treatment of moderate-to-severe atopic dermatitis: results from the double-blind, randomized, multicentre, placebo-controlled phase III ECZTRA 3 trial. $\mathrm{Br} J$ Dermatol. 2021;184:450-463. doi:10.1111/bjd.19573

17. Papp K, Szepietowski JC, Kircik L, et al. Efficacy and safety of ruxolitinib cream for the treatment of atopic dermatitis: results from 2 phase 3, randomized, double-blind studies [published online May 4, 2021]. J Am Acad Dermatol. doi:10.1016/j.jaad.2021.04.085

18. Paller AS, Stein Gold L, Soung J, et al. Efficacy and patientreported outcomes from a phase $2 \mathrm{~b}$, randomized clinical trial of tapinarof cream for the treatment of adolescents and adults with atopic dermatitis. J Am Acad Dermatol. 2021;84:632-638. doi:10.1016/j .jaad.2020.05.135

19. Nakatsuji, T, Hata TR, Tong $Y$, et al. Development of a human skin commensal microbe for bacteriotherapy of atopic dermatitis and use in a phase 1 randomized clinical trial [published online February 22, 2021]. Nat Med. 2021;27:700-709. doi:10.1038/s41591-021-01256-2

20. Kong $\mathrm{HH}, \mathrm{Oh}$ J, Deming $\mathrm{C}$, et al. Temporal shifts in the skin microbiome associated with disease flares and treatment in children with atopic dermatitis. Genome Res. 2012;22:850-859. doi:10.1101 Igr.131029.111

21. Myles IA, Castillo CR, Barbian KD, et al. Therapeutic responses to Roseomonas mucosa in atopic dermatitis may involve lipidmediatedTNF-related epithelial repair. Sci Transl Med. 2020;12:eaaz8631. doi:10.1126/scitranslmed.aaz8631

22. Mortz CG, Lauritsen JM, Bindslev-Jensen C, et al. Prevalence of atopic dermatitis, asthma, allergic rhinitis, and hand and contact dermatitis in adolescents. The Odense Adolescence Cohort Study on Atopic Diseases and Dermatitis. Br J Dermatol. 2001;144:523-532. doi:10.1046/j.1365-2133.2001.04078.x

23. Grönhagen $\mathrm{C}$, Lidén $\mathrm{C}$, Wahlgren $\mathrm{CF}$, et al. Hand eczema and atopic dermatitis in adolescents: a prospective cohort study from the BAMSE project. Br J Dermatol. 2015;173:1175-1182. doi:10.1111/bjd.14019

24. Mortz CG, Lauritsen JM, Bindslev-Jensen C, et al. Contact allergy and allergic contact dermatitis in adolescents: prevalence measures and associations. The Odense Adolescence Cohort Study on Atopic Diseases and Dermatitis (TOACS). Acta Derm Venereol. 2002;82:352-358. doi:10.1080/000155502320624087

25. Isaksson $\mathrm{M}$, Olhardt $\mathrm{S}$, Rådehed J, et al. Children with atopic dermatitis should always be patch-tested if they have hand or foot dermatitis. Acta Derm Venereol. 2015;95:583-586. doi:10.2340/00015555-1995

26. Silverberg JI, Warshaw EM, Maibach HI, et al. Hand eczema in children referred for patch testing: North American Contact Dermatitis Group Data, 2000-2016. Br J Dermatol. 2021;185:185-194. doi:10.1111/bjd.19818

27. Agner T, Elsner P. Hand eczema: epidemiology, prognosis and prevention. J Eur Acad Dermatol Venereol. 2020;34(suppl 1):4-12. doi:10.1111/jdv.16061

28. Cazzaniga S, Ballmer-Weber BK, Gräni N, et al. Medical, psychological and socio-economic implications of chronic hand eczema: a crosssectional study. I Eur Acad Dermatol Venereol. 2016;30:628-637. doi:10.1111/jdv.13479 